EUROPEAN LABORATORY FOR PARTICLE PHYSICS (CERN)

CERN-PPE/95-023

2 March, 1995

\title{
Improved Tau Polarisation Measurement
}

\author{
The ALEPH Collaboration*
}

\begin{abstract}
Using $22 \mathrm{pb}^{-1}$ of data collected at LEP in 1992 on the peak of the $\mathrm{Z}$ resonance, the ALEPH collaboration has measured the polarisation of the tau leptons decaying into $e \nu \bar{\nu}, \mu \nu \bar{\nu}, \pi \nu, \rho \nu$ and $a_{1} \nu$ from their individual decay product distributions. The measurement of the tau polarisation as a function of the production polar angle yields the two parameters $\mathcal{A}_{\tau}$ and $\mathcal{A}_{e}$, where, in terms of the axial and vector couplings $g_{A l}$ and $g_{V l}, \mathcal{A}_{l}=2 g_{V l} g_{A l} /\left(g_{V l}^{2}+g_{A l}^{2}\right)$. This analysis follows to a large extent the methods devised for the 1990 and 1991 data but with improvements which bring a better understanding of the systematic uncertainties. Combining the 1992 measurements with our previously published results yields $\mathcal{A}_{\tau}=0.136 \pm 0.012 \pm 0.009$ and $\mathcal{A}_{e}=0.129 \pm 0.016 \pm 0.005$. Assuming $e-\tau$ universality, the measurements imply an effective weak mixing angle of $\sin ^{2} \theta_{W}^{e f f}=0.2332 \pm 0.0014$.
\end{abstract}

(Submitted to Zeitschrift für Physik.)

* See the following pages for the list of authors. 


\section{The ALEPH Collaboration}

D. Buskulic, D. Casper, I. De Bonis, D. Decamp, P. Ghez, C. Goy, J.-P. Lees, M.-N. Minard, P. Odier, B. Pietrzyk

Laboratoire de Physique des Particules (LAPP), IN ${ }^{2} P^{3}$-CNRS, 74019 Annecy-le-Vieux Cedex, France

F. Ariztizabal, M. Chmeissani, J.M. Crespo, I. Efthymiopoulos, E. Fernandez, M. Fernandez-Bosman, V. Gaitan, Ll. Garrido, ${ }^{15}$ M. Martinez, S. Orteu, A. Pacheco, C. Padilla, F. Palla, A. Pascual, J.A. Perlas, F. Sanchez, F. Teubert

Institut de Fisica d'Altes Energies, Universitat Autonoma de Barcelona, 08193 Bellaterra (Barcelona), $\operatorname{Spain}^{7}$

D. Creanza, M. de Palma, A. Farilla, G. Iaselli, G. Maggi, ${ }^{3}$ N. Marinelli, S. Natali, S. Nuzzo, A. Ranieri, G. Raso, F. Romano, F. Ruggieri, G. Selvaggi, L. Silvestris, P. Tempesta, G. Zito

Dipartimento di Fisica, INFN Sezione di Bari, 70126 Bari, Italy

X. Huang, J. Lin, Q. Ouyang, T. Wang, Y. Xie, R. Xu, S. Xue, J. Zhang, L. Zhang, W. Zhao

Institute of High-Energy Physics, Academia Sinica, Beijing, The People's Republic of China ${ }^{8}$

G. Bonvicini, M. Cattaneo, P. Comas, P. Coyle, H. Drevermann, A. Engelhardt, R.W. Forty, M. Frank, M. Girone, R. Hagelberg, J. Harvey, R. Jacobsen, ${ }^{24}$ P. Janot, B. Jost, J. Knobloch, I. Lehraus, M. Maggi, C. Markou, ${ }^{27}$ E.B. Martin, P. Mato, H. Meinhard, A. Minten, R. Miquel, T. Oest, P. Palazzi, J.R. Pater, P. Perrodo, J.-F. Pusztaszeri, F. Ranjard, P. Rensing, L. Rolandi, D. Schlatter, M. Schmelling, O. Schneider, W. Tejessy, I.R. Tomalin, A. Venturi, H. Wachsmuth, W. Wiedenmann, T. Wildish, W. Witzeling, J. Wotschack

European Laboratory for Particle Physics (CERN), 1211 Geneva 23, Switzerland

Z. Ajaltouni, M. Bardadin-Otwinowska, ${ }^{2}$ A. Barres, C. Boyer, A. Falvard, P. Gay, C. Guicheney, P. Henrard, J. Jousset, B. Michel, S. Monteil, J-C. Montret, D. Pallin, P. Perret, F. Podlyski, J. Proriol, J.-M. Rossignol, F. Saadi

Laboratoire de Physique Corpusculaire, Université Blaise Pascal, IN ${ }^{2} P^{3}$-CNRS, Clermont-Ferrand, 63177 Aubière, France

T. Fearnley, J.B. Hansen, J.D. Hansen, J.R. Hansen, P.H. Hansen, B.S. Nilsson

Niels Bohr Institute, 2100 Copenhagen, Denmark ${ }^{9}$

A. Kyriakis, E. Simopoulou, I. Siotis, A. Vayaki, K. Zachariadou

Nuclear Research Center Demokritos (NRCD), Athens, Greece

A. Blondel, ${ }^{21}$ G. Bonneaud, J.C. Brient, P. Bourdon, L. Passalacqua, A. Rougé, M. Rumpf, R. Tanaka, A. Valassi, M. Verderi, H. Videau

Laboratoire de Physique Nucléaire et des Hautes Energies, Ecole Polytechnique, IN ${ }^{2} P^{3}-C N R S, 91128$ Palaiseau Cedex, France 
D.J. Candlin, M.I. Parsons

Department of Physics, University of Edinburgh, Edinburgh EH9 3JZ, United Kingdom ${ }^{10}$

E. Focardi, G. Parrini

Dipartimento di Fisica, Università di Firenze, INFN Sezione di Firenze, 50125 Firenze, Italy

M. Corden, M. Delfino, ${ }^{12}$ C. Georgiopoulos, D.E. Jaffe

Supercomputer Computations Research Institute, Florida State University, Tallahassee, FL 323064052, USA 13,14

A. Antonelli, G. Bencivenni, G. Bologna, ${ }_{4}^{4}$ F. Bossi, P. Campana, G. Capon, F. Cerutti, V. Chiarella, G. Felici, P. Laurelli, G. Mannocchi, ${ }^{5}$ F. Murtas, G.P. Murtas, M. Pepe-Altarelli

Laboratori Nazionali dell'INFN (LNF-INFN), 00044 Frascati, Italy

S.J. Dorris, A.W. Halley, I. ten Have, ${ }^{6}$ I.G. Knowles, J.G. Lynch, W.T. Morton, V. O’Shea, C. Raine, P. Reeves, J.M. Scarr, K. Smith, M.G.Smith, A.S. Thompson, F. Thomson, S. Thorn, R. M. Turnbull

Department of Physics and Astronomy, University of Glasgow, Glasgow G12 8QQ,United Kingdom ${ }^{10}$

U. Becker, O. Braun, C. Geweniger, G. Graefe, P. Hanke, V. Hepp, E.E. Kluge, A. Putzer, B. Rensch, M. Schmidt, J. Sommer, H. Stenzel, K. Tittel, M. Wunsch

Institut für Hochenergiephysik, Universität Heidelberg, 69120 Heidelberg, Fed. Rep. of Germany ${ }^{16}$

R. Beuselinck, D.M. Binnie, W. Cameron, D.J. Colling, P.J. Dornan, N. Konstantinidis, L. Moneta, A. Moutoussi, J. Nash, G. San Martin, J.K. Sedgbeer, A.M. Stacey

Department of Physics, Imperial College, London SW7 2BZ, United Kingdom ${ }^{10}$

G. Dissertori, P. Girtler, E. Kneringer, D. Kuhn, G. Rudolph

Institut für Experimentalphysik, Universität Innsbruck, 6020 Innsbruck, Austria ${ }^{18}$

C.K. Bowdery, T.J. Brodbeck, P. Colrain, G. Crawford, A..J. Finch, F. Foster, G. Hughes, T. Sloan, E.P. Whelan, M.I. Williams

Department of Physics, University of Lancaster, Lancaster LA1 4YB, United Kingdom ${ }^{10}$

A. Galla, A.M. Greene, K. Kleinknecht, G. Quast, J. Raab, B. Renk, H.-G. Sander, R. Wanke, C. Zeitnitz

Institut für Physik, Universität Mainz, 55099 Mainz, Fed. Rep. of Germany ${ }^{16}$

J.J. Aubert, A.M. Bencheikh, C. Benchouk, A. Bonissent, G. Bujosa, D. Calvet, J. Carr, C. Diaconu, F. Etienne, M. Thulasidas, D. Nicod, P. Payre, D. Rousseau, M. Talby

Centre de Physique des Particules, Faculté des Sciences de Luminy, IN ${ }^{2} P^{3}$-CNRS, 13288 Marseille, France 
I. Abt, R. Assmann, C. Bauer, W. Blum, D. Brown, ${ }^{24}$ H. Dietl, F. Dydak, ${ }^{21}$ C. Gotzhein, K. Jakobs, H. Kroha, G. Lütjens, G. Lutz, W. Männer, H.-G. Moser, R. Richter, A. Rosado-Schlosser, A.S. Schwarz, ${ }^{23}$ R. Settles, H. Seywerd, U. Stierlin, ${ }^{2}$ R. St. Denis, G. Wolf

Max-Planck-Institut für Physik, Werner-Heisenberg-Institut, 80805 München, Fed. Rep. of Germany ${ }^{16}$

R. Alemany, J. Boucrot, O. Callot, A. Cordier, F. Courault, M. Davier, L. Duflot, J.-F. Grivaz, Ph. Heusse, M. Jacquet, D.W. Kim, ${ }^{19}$ F. Le Diberder, J. Lefrançois, A.-M. Lutz, G. Musolino, I. Nikolic, H.J. Park, I.C. Park, M.-H. Schune, S. Simion, J.-J. Veillet, I. Videau

Laboratoire de l'Accélérateur Linéaire, Université de Paris-Sud, IN ${ }^{2} P^{3}-C N R S, 91405$ Orsay Cedex, France

P. Azzurri, D. Abbaneo, G. Bagliesi, G. Batignani, S. Bettarini, C. Bozzi, G. Calderini, M. Carpinelli, M.A. Ciocci, V. Ciulli, R. Dell'Orso, I. Ferrante, L. Foà, ${ }^{1}$ F. Forti, A. Giassi, M.A. Giorgi, A. Gregorio, F. Ligabue, A. Lusiani, P.S. Marrocchesi, A. Messineo, G. Rizzo, G. Sanguinetti, A. Sciabà, P. Spagnolo, J. Steinberger, R. Tenchini, G. Tonelli, ${ }^{26}$ G. Triggiani, C. Vannini, P.G. Verdini, J. Walsh

Dipartimento di Fisica dell'Università, INFN Sezione di Pisa, e Scuola Normale Superiore, 56010 Pisa, Italy

A.P. Betteridge, G.A. Blair, L.M. Bryant, Y. Gao, M.G. Green, D.L. Johnson, T. Medcalf, Ll.M. Mir, J.A. Strong

Department of Physics, Royal Holloway \& Bedford New College, University of London, Surrey TW20 OEX, United Kingdom ${ }^{10}$

V. Bertin, D.R. Botterill, R.W. Clifft, T.R. Edgecock, S. Haywood, M. Edwards, P. Maley, P.R. Norton, J.C. Thompson

Particle Physics Dept., Rutherford Appleton Laboratory, Chilton, Didcot, Oxon OX11 OQX, United Kingdom ${ }^{10}$

B. Bloch-Devaux, P. Colas, H. Duarte, S. Emery, W. Kozanecki, E. Lançon, M.C. Lemaire, E. Locci, B. Marx, P. Perez, J. Rander, J.-F. Renardy, A. Rosowsky, A. Roussarie, J.-P. Schuller, J. Schwindling, D. Si Mohand, A. Trabelsi, B. Vallage

CEA, DAPNIA/Service de Physique des Particules, CE-Saclay, 91191 Gif-sur-Yvette Cedex, France ${ }^{17}$

R.P. Johnson, A.M. Litke, G. Taylor, J. Wear

Institute for Particle Physics, University of California at Santa Cruz, Santa Cruz, CA 95064, USA 22

A. Beddall, C.N. Booth, R. Boswell, S. Cartwright, F. Combley, I. Dawson, A. Koksal, M. Letho, W.M. Newton, C. Rankin, L.F. Thompson

Department of Physics, University of Sheffield, Sheffield S3 $7 R H$, United Kingdom ${ }^{10}$ 
A. Böhrer, S. Brandt, G. Cowan, E. Feigl, C. Grupen, G. Lutters, J. Minguet-Rodriguez, F. Rivera, ${ }^{25}$ P. Saraiva, L. Smolik, F. Stephan

Fachbereich Physik, Universität Siegen, 57068 Siegen, Fed. Rep. of Germany ${ }^{16}$

L. Bosisio, R. Della Marina, G. Ganis, G. Giannini, B. Gobbo, L. Pitis, F. Ragusa ${ }^{20}$

Dipartimento di Fisica, Università di Trieste e INFN Sezione di Trieste, 34127 Trieste, Italy

H. Kim, J. Rothberg, S. Wasserbaech

Experimental Elementary Particle Physics, University of Washington, WA 98195 Seattle, U.S.A.

S.R. Armstrong, L. Bellantoni, P. Elmer, Z. Feng, D.P.S. Ferguson, Y.S. Gao, S. González, J. Grahl, J.L. Harton, O.J. Hayes, H. Hu, P.A. McNamara III, J.M. Nachtman, W. Orejudos, Y.B. Pan, Y. Saadi, M. Schmitt, I.J. Scott, V. Sharma, J.D. Turk, A.M. Walsh, F.V. Weber, ${ }^{1}$ Sau Lan Wu, X. Wu, J.M. Yamartino, M. Zheng, G. Zobernig

Department of Physics, University of Wisconsin, Madison, WI 53706, USA ${ }^{11}$

\footnotetext{
${ }^{1}$ Now at CERN, 1211 Geneva 23, Switzerland.

${ }^{2}$ Deceased.

${ }^{3}$ Now at Dipartimento di Fisica, Università di Lecce, 73100 Lecce, Italy.

${ }^{4}$ Also Istituto di Fisica Generale, Università di Torino, Torino, Italy.

${ }^{5}$ Also Istituto di Cosmo-Geofisica del C.N.R., Torino, Italy.

${ }^{6}$ Now at TSM Business School, Enschede, The Netherlands.

${ }^{7}$ Supported by CICYT, Spain.

${ }^{8}$ Supported by the National Science Foundation of China.

${ }^{9}$ Supported by the Danish Natural Science Research Council.

${ }^{10}$ Supported by the UK Particle Physics and Astronomy Research Council.

${ }^{11}$ Supported by the US Department of Energy, contract DE-AC02-76ER00881.

${ }^{12}$ On leave from Universitat Autonoma de Barcelona, Barcelona, Spain.

${ }^{13}$ Supported by the US Department of Energy, contract DE-FG05-92ER40742.

${ }^{14}$ Supported by the US Department of Energy, contract DE-FC05-85ER250000.

${ }^{15}$ Permanent address: Universitat de Barcelona, 08208 Barcelona, Spain.

${ }^{16}$ Supported by the Bundesministerium für Forschung und Technologie, Fed. Rep. of Germany.

${ }^{17}$ Supported by the Direction des Sciences de la Matière, C.E.A.

${ }^{18}$ Supported by Fonds zur Förderung der wissenschaftlichen Forschung, Austria.

${ }^{19}$ Permanent address: Kangnung National University, Kangnung, Korea.

${ }^{20}$ Now at Dipartimento di Fisica, Università di Milano, Milano, Italy.

${ }^{21}$ Also at CERN, 1211 Geneva 23, Switzerland.

${ }^{22}$ Supported by the US Department of Energy, grant DE-FG03-92ER40689.

${ }^{23}$ Now at DESY, Hamburg, Germany.

${ }^{24}$ Now at Lawrence Berkeley Laboratory, Berkeley, CA 94720, USA.

${ }^{25}$ Partially supported by Colciencias, Colombia.

${ }^{26} \mathrm{Also}$ at Istituto di Matematica e Fisica, Università di Sassari, Sassari, Italy.

${ }^{27}$ Now at University of Athens, 157-71 Athens, Greece.
} 


\section{Introduction}

Parity violation in $\mathrm{Z}$ production via $e^{+} e^{-}$annihilation at centre of mass energies around the $\mathrm{Z}$ resonance is manifest in the difference in couplings of the $\mathrm{Z}$ to right-handed and left-handed electrons and positrons. Similarly parity violation causes leptons from Z decay to be polarised. The $\mathrm{Z}$ and lepton polarisations can be measured if the average helicity of the final-state leptons is determined as a function of the polar angle. Such measurements are possible with the tau at LEP since the tau's decay products are measurable. The study of tau polarisation at the $\mathrm{Z}$ is now a well established part of the electroweak physics programme at LEP $[1,2,3]$.

The helicities of the two taus from $Z$ decay are nearly $100 \%$ anti-correlated [4] and the tau polarisation is defined as

$$
P_{\tau}=\frac{\sigma_{R}-\sigma_{L}}{\sigma_{R}+\sigma_{L}}
$$

where $\sigma_{R}$ is the cross section to produce a right-handed $\tau^{-}$along with a left-handed $\tau^{+}$, and $\sigma_{L}$ is the cross section to produce a left-handed $\tau^{-}$and a right-handed $\tau^{+}$. The tau polarisation as a function of polar angle $\theta$, defined as the angle between the directions of the $e^{-}$beam and the $\tau^{-}$, is given to a good approximation near the $\mathrm{Z}$ resonance by $[5]$

$$
P_{\tau}(\cos \theta)=-\frac{\mathcal{A}_{\tau}\left(1+\cos ^{2} \theta\right)+\mathcal{A}_{e}(2 \cos \theta)}{\left(1+\cos ^{2} \theta\right)+\mathcal{A}_{\tau} \mathcal{A}_{e}(2 \cos \theta)} .
$$

The asymmetry parameters $\mathcal{A}_{l}$ are given in terms of the effective vector and axial vector couplings of the $Z$ to the lepton $l, g_{V l}$ and $g_{A l}$, by

$$
\mathcal{A}_{l}=\frac{2 g_{V l} g_{A l}}{\left(g_{V l}^{2}+g_{A l}^{2}\right)}
$$

The effective weak mixing angle $\theta_{W}^{e f f}$ is defined by

$$
\sin ^{2} \theta_{W}^{e f f}=\frac{1}{4}\left(1-\frac{g_{V l}}{g_{A l}}\right) .
$$

This article presents an update to the tau polarisation measurement by the ALEPH Collaboration. The new data analyzed were recorded in 1992 and represent an integrated luminosity of $22 \mathrm{pb}^{-1}$, all taken at the peak of the $\mathrm{Z}$ resonance (average centre of mass energy $91.274 \mathrm{GeV}$ ). The five tau decay modes $e \nu \bar{\nu}, \mu \nu \bar{\nu}, \pi \nu, \rho \nu$, and $a_{1} \nu$ have been used to extract the electron and tau couplings to the $Z$. The analysis is very similar to the single-tau method published in [2]. The polarisation is inferred for each of the five decay channels from the distribution of the variable which summarises all the information available from a single tau decay for that decay mode. For the electron, muon, and pion channels this variable is the ratio of the particle energy to the beam energy, written $x$. In the case of the $\rho$ and the $a_{1}$ the $\omega$ variable, described in [2], is used $^{1}$. In essence $\omega$ is the asymmetry of the densities for right-handed and left-handed

\footnotetext{
${ }^{1}$ There is an overall sign error in the second equation of formula 31 of [2]. The left-hand side of the equation should read $w_{-1}^{-}-w_{1}^{-}$.
} 
taus in the space of the variables which fully describe the tau decay. To extract the polarisation a linear combination of the simulated [6] $x$ or $\omega$ distributions from the taus contributing to $\sigma_{R}$ and $\sigma_{L}$ along with the estimated non-tau background, is fit to the data for each channel.

This work presents new understanding of some systematic effects and hence reduced systematic uncertainties.

\section{The ALEPH Detector}

The ALEPH detector has been described in detail elsewhere [7]. This analysis uses almost all the subcomponents of the detector: the silicon microstrip vertex detector, the cylindrical eight-layer inner tracking drift chamber, the 1.8 meter radius time projection chamber (TPC), the lead/proportional chamber electromagnetic calorimeter (ECAL), the iron/streamer chamber hadron calorimeter (HCAL), and the two double layers of muon chambers outside the hadron calorimeter.

The tracking chambers and the ECAL are all immersed in a $1.5 \mathrm{~T}$ axial magnetic field; the HCAL also serves as the flux return. Details of how these subdetectors are exploited for the tau polarisation measurement may be found in reference [2].

In view of its importance in the $e \nu \bar{\nu}$ and $\rho \nu$ channels, the ECAL readout is described in more detail. The ECAL is read out in two ways: through the anode wires, summed over each of the 36 mechanical modules, and through projective $0.8^{\circ} \times 0.8^{\circ}$ cathode towers with three segments in depth of 4,9 , and 9 radiation lengths. Each of the 220,000 tower segments has an energy threshold of about $30 \mathrm{MeV}$. The wires have a similar threshold, but on a whole mechanical module.

\section{Particle Identification}

\subsection{Charged particle identification}

As in the previously published work two analysis methods, which differ mainly in charged particle identification, have been used. One particle identification algorithm uses the neural network (NN method) described in [2], the other uses a likelihood technique (LM method) which has evolved from the CC method, which used cuts and is discussed in the same reference. In the LM method cuts are made on probabilities derived from Monte Carlo likelihood distributions which are built for a set of discriminating variables. The variables are grouped in subsets ordered by decreasing discriminating power and the particle identity, between electron and pion or muon and pion, is decided as soon as the likelihood for the examined variables is good enough. The method improves on the old one, in particular for electron-pion separation.

For both methods electron-pion separation is performed mainly with information from the $\mathrm{d} E / \mathrm{d} x$ measurement in the TPC and shower energy and shape in the ECAL. The muon chambers and the HCAL digital response are the main tools for muon-pion separation. 
The efficiencies for particle identification are checked on samples of electrons, muons, and pions from data and Monte Carlo which are selected using information other than the particle identification for the particle in question. These particles will be called "kinematically identified". The kinematically identified electron sample con-

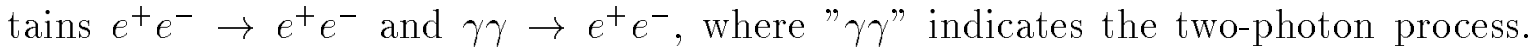
The muon sample is composed of $e^{+} e^{-} \rightarrow \mu^{+} \mu^{-}$and $\gamma \gamma \rightarrow \mu^{+} \mu^{-}$. To select the kinematically identified leptons the track in one hemisphere is identified as an electron or a muon, then cuts are applied only on particle momentum or energy to determine if the track in the opposite hemisphere may become part of the sample. For example, consider events with two back-to-back tracks each with energy near the beam energy; if the track in one hemisphere is identified as a muon then the track in the other hemisphere is also a muon more than $99 \%$ of the time. Kinematically identified pions are provided by $\tau \rightarrow \rho \nu$ with a well identified $\pi^{0}$ and a $\pi-\pi^{0}$ mass in the range 0.5 to 1.2 $\mathrm{GeV}$. Three-prong tau decays are used as well: one track is put in the pion sample if neither of the other two is identified as an electron.

Table 1 shows the charged particle identification efficiencies obtained on $\tau^{+} \tau^{-}$ Monte Carlo for an angular range $-0.9<\cos \theta<0.9$ and for a ratio of the reconstructed momentum to the beam energy in the range $0.05<p / E_{\text {beam }}<1$. . In the LM method, for electron-pion separation the tracks are required not to go into the insensitive spaces, called cracks, between ECAL modules; this fiducial requirement introduces a $4 \%$ loss in acceptance.

\begin{tabular}{|l|c|c|c|c|}
\hline $\begin{array}{l}\tau \text { decays } \rightarrow \\
\text { identified as } \downarrow\end{array}$ & e $\nu \nu$ & $\mu \nu \nu$ & $\pi \nu$ & $\pi+n \pi^{0} \nu$ \\
\hline electron & $\mathbf{9 9 . 5 / 9 8 . 8}$ & $0.0 / 0.0$ & $0.5 / 1.2$ & $0.8 / 1.3$ \\
\hline muon & $0.0 / 0.0$ & $\mathbf{9 9 . 7 / 9 9 . 2}$ & $1.0 / 1.4$ & $1.4 / 1.7$ \\
\hline pion & $0.5 / 1.2$ & $0.3 / 0.8$ & $\mathbf{9 8 . 5} / \mathbf{9 7 . 4}$ & $\mathbf{9 7 . 7} / \mathbf{9 7 . 0}$ \\
\hline
\end{tabular}

Table 1: Probabilities to be identified as electron, muon or pion for electrons, muons or pions produced in different $\tau$ decays. The numbers come from Monte Carlo. The first number corresponds to the LM, the second to the NN method.

To estimate the systematic uncertainties induced by particle identification, the identification methods are checked on the samples of kinematically identified particles. Any momentum dependent differences in identification efficiencies between the data and the full detector simulation Monte Carlo will affect the polarisation fit. The systematic uncertainties on the polarisation measurement due to particle identification are shown in Table 2. For the channels $e \nu \bar{\nu}, \mu \nu \bar{\nu}$, and $\pi \nu$ they are extracted from the ratios of the identification efficiencies in data and Monte Carlo as a function of momentum. The same is done in the $\rho \nu$ and $a_{1} \nu$ channels, but as a function of $\omega$. A possible dependence on momentum in the pion channel is corrected. The polarisation biases found in the electron and muon channels are much smaller than their uncertainties and are left uncorrected.

Similarly, misidentification ratios (e.g., for pions misidentified as electrons) enter the 
systematic uncertainties on tau and non-tau backgrounds. The overall identification efficiencies in data and in Monte Carlo are found to agree for electrons and muons. A $(1.0 \pm 0.1) \%$ difference between data and Monte Carlo in the overall pion efficiency in the LM method, which has also been observed in earlier study [2], is corrected for.

\subsection{Photon and $\pi^{0}$ identification}

Reconstruction of photons, in the ECAL and from $e^{+} e^{-}$conversion pairs, follows closely the methods described in [2]. Photon reconstruction in the ECAL starts from local energy maxima in the projective towers and neighboring deposits are added to form photon candidates.

The energy deposit of a charged track in the ECAL may produce local energy maxima which are interpreted by the algorithm as photons. Real photons usually deposit more than $70 \%$ of their energy in the first two depth segments of the ECAL. Requiring this for all ECAL photons eliminates many "fake" photons. Furthermore, fake photons are generally close $(<2.5 \mathrm{~cm})$ to a track impact point on the ECAL and lower than about $500 \mathrm{MeV}$ in energy. Their number is further reduced by cutting in the $(\mathrm{E}, \mathrm{d})$ plane, where $\mathrm{E}$ is the photon energy and $\mathrm{d}$ its distance to the closest point of impact of a charged track on ECAL.

Candidate $\pi^{0}$ 's may be reconstructed from a system of two photons with mass in the range $0.08<M_{\gamma \gamma}<0.20 \mathrm{MeV}$. These are referred to as "resolved" $\pi^{0}$ 's. About $61 \%$ of the final $\tau \rightarrow \rho \nu$ candidates have a resolved $\pi^{0}$. As is often the case in the $\rho$ channel, the two photons from the $\pi^{0}$ may merge in the ECAL and only one local energy maximum, and hence only one photon candidate, is formed. One of the photons from a $\pi^{0}$ may also be lost in a crack or have energy below the $250 \mathrm{MeV}$ threshold. Single-photon candidates are accepted as $\pi^{0}$ 's if their energy is above $3 \mathrm{GeV}$, and they are called "merged" $\pi^{0}$ 's. According to $\tau^{+} \tau^{-}$event simulation about $75 \%$ of the merged $\pi^{0}$ s contain the two photons from a $\pi^{0}$.

\section{Polarisation with Individual Channels}

\subsection{Introduction}

The two analyses do not have identical selection procedures and they are independently optimised. They are, however, similar enough to be described together. The selection follows three steps, first a preselection reduces the $Z \rightarrow q \bar{q}$ background, keeping virtually all the $Z \rightarrow l^{+} l^{-}$events. The preselection retains events with between 2 and 8 charged tracks and requires $|\cos \theta|<0.9$, where $\theta$ is the angle of the event thrust axis, calculated with charged tracks only, with respect to the beam axis. The preselection also requires the presence of at least one charged track or photon of energy greater than $2 \mathrm{GeV}$.

The selected events are analysed in two halves, called hemispheres, each containing the decay products of one tau. In the second step each hemisphere is classified 


\begin{tabular}{|l|c|c|c|c|c|}
\hline & $e \nu \bar{\nu}$ & $\mu \nu \bar{\nu}$ & $\pi \nu$ & $\rho \nu$ & $a_{1} \nu$ \\
\hline charged particle id. & 0.4 & 0.4 & $0.4 / 0.6$ & 0.1 & $0.4 / 0.3$ \\
\hline$\pi^{0}$ identification & - & - & - & 1.3 & 0.2 \\
\hline tracking & - & - & - & - & 0.5 \\
\hline$\tau$ background & $0.5 / 1.3$ & $0.5 / 0.7$ & 0.5 & 0.1 & $0.8 / 0.3$ \\
\hline non- $\tau$ background & $2.0 / 0.6$ & $1.1 / 0.8$ & 0.2 & 0.2 & - \\
\hline energy calibration & $1.0 / 3.5$ & 1.0 & 0.1 & 0.7 & 0.1 \\
\hline model & - & - & - & - & 1.2 \\
\hline Monte Carlo statistic & $2.2 / 2.5$ & $1.7 / 1.9$ & 0.9 & 0.8 & 1.5 \\
\hline total & $3.2 / 4.6$ & $2.3 / 2.4$ & $1.1 / 1.2$ & 1.7 & $2.2 / 2.1$ \\
\hline
\end{tabular}

Table 2: Systematic uncertainties (times $10^{2}$ ) in the individual channel measurements of the average polarisation for the 1992 data. When two numbers are quoted the first is for the LM analysis and the second is for the NN analysis.

as a candidate for a studied decay channel or is left unclassified. The high efficiency of the charged particle identification allows for simple and efficient channel classification. Finally, the non- $\tau$ background is reduced by setting conditions on the recoil hemisphere or on correlations between hemispheres. Background from $\gamma \gamma \rightarrow \tau^{+} \tau^{-}$and $\gamma \gamma \rightarrow$ hadrons is then negligible in all decay channels.

Table 3 summarises the acceptances and contaminations in the five individual channels for the 1992 data. Figures 1 and 2 show the distributions of the polarisation sensitive variables for the data with the results of the Monte Carlo simulation fits.

\begin{tabular}{|r|r|r|r|r|r|}
\hline Decay channel & $e \nu \nu$ & $\mu \nu \nu$ & $\pi \nu$ & $\rho \nu$ & $a_{1} \nu$ \\
\hline Candidates (1992 data) & 6141 & 9020 & 5229 & 9265 & 3800 \\
& 5836 & 8208 & 5062 & 8256 & 3477 \\
\hline Fit range: x for $e \nu \bar{\nu}, \mu \nu \bar{\nu}, \pi \nu$ & {$[0.0,1.1]$} & {$[0.05,1.15]$} & {$[0.05,1.15]$} & {$[-1,1]$} & {$[-1,1]$} \\
$\omega$ for $\rho \nu$ and $a_{1} \nu$ & {$[0.0,1.0]$} & {$[0.066,0.95]$} & {$[0.066,1.0]$} & {$[-1,1]$} & {$[-1,1]$} \\
\hline Acceptance(\%) & 50 & 74 & $* 61$ & $* 52$ & 56 \\
& 48 & 69 & 60 & 47 & 52 \\
\hline Tau background(\%) & 1.2 & $* 2.2$ & 5.8 & 8.4 & 8.9 \\
& 2.0 & 1.3 & 7.0 & 7.0 & 8.3 \\
\hline Non tau-background(\%) & 0.5 & 1.4 & 0.8 & 0.1 & 0.0 \\
& 1.4 & 1.0 & 1.1 & 0.2 & 0.1 \\
\hline
\end{tabular}

Table 3: Acceptances and contaminations in the five channels for the 1992 data. The quantities with an * are obtained using the particle identification efficiencies measured on data, when observed to be different from the ones measured on Monte Carlo by more than one standard deviation. The first line is for LM, the second for NN.

Table 4 shows the results for the LM and NN methods as well as the difference between them due to the non-common sample of events. The last column of table 4 
shows the results of combining the two analyses assuming that their systematic uncertainties are fully correlated. Discussion of the estimation of systematic uncertainties are given channel by channel in the following sections. The systematic uncertainties are summarized in table 2. Explanation of the most important parts of the channel classification and background rejection are given in the following sections.

\begin{tabular}{|l|r|r|r||r|}
\hline & LM & NN & difference & 1992 ALEPH result \\
\hline$e \nu \nu$ & $-0.177 \pm 0.064$ & $-0.233 \pm 0.071$ & $0.056 \pm 0.044$ & $-0.187 \pm 0.063 \pm 0.033$ \\
\hline$\mu \nu \nu$ & $-0.105 \pm 0.052$ & $-0.111 \pm 0.056$ & $0.006 \pm 0.025$ & $-0.106 \pm 0.052 \pm 0.023$ \\
\hline$\pi \nu$ & $-0.147 \pm 0.026$ & $-0.150 \pm 0.028$ & $0.003 \pm 0.016$ & $-0.148 \pm 0.026 \pm 0.011$ \\
\hline$\rho \nu$ & $-0.097 \pm 0.024$ & $-0.086 \pm 0.025$ & $-0.011 \pm 0.013$ & $-0.092 \pm 0.024 \pm 0.017$ \\
\hline$a_{1} \nu$ & $-0.155 \pm 0.043$ & $-0.129 \pm 0.044$ & $-0.026 \pm 0.022$ & $-0.143 \pm 0.042 \pm 0.021$ \\
\hline
\end{tabular}

Table 4: Average polarisation and statistical uncertainty for the five channels from the two analyses (called LM and NN) for the 1992 data only. The difference is shown in the third column and its uncertainty is calculated using only the statistical overlap between the two analyses. The last column shows the ALEPH result for the 1992 data, calculated by combining the LM and NN analyses assuming the two methods have fully correlated systematic uncertainties.

\subsection{Polarisation with $e \nu \bar{\nu}$}

An electron candidate hemisphere has one track identified as an electron and any number of photons as long as no two form a $\pi^{0}$ mass. To reduce background from $e^{+} e^{-} \rightarrow e^{+} e^{-}$the leading track in the opposite hemisphere must not be an electron and it must have momentum less than $90 \%(75 \%)$ of the beam energy for the LM (NN) analysis. If the leading track in the opposite hemisphere is identified as a pion with momentum above $30 \%$ of the beam energy and it goes in a crack between ECAL modules then the candidate hemisphere is rejected by the NN analysis. The LM analysis rejects any candidate with the most energetic track in the opposite hemisphere going into an ECAL crack, unless that track is identified as a muon. Two photon background is reduced by rejecting events with acollinearity less than $160^{\circ}$. The range of polar angle $\theta$ corresponding to the region of overlap between the barrel and end cap calorimeters (about $0.7<|\cos \theta|<0.8$ ) is excluded by both analyses due to difficulties in measuring the electron energy there.

To estimate the electron energy both LM and NN use the ECAL. The calorimetric energy measurements include all radiated photons. This permits retention of full statistical precision on the polarisation and avoids systematic effects which might result from imperfectly simulated radiation. The LM analysis uses the energy deposit as measured on the wires of the ECAL while the NN method uses the energy deposit measured on the projective towers of the ECAL. When the candidate track enters a crack between ECAL modules the NN analysis uses the momentum of the track plus the energy of any photons in the hemisphere as the electron energy estimator. Such 
hemispheres are rejected by the LM analysis. Using the track momentum plus the photons as the electron energy estimator for all electron hemispheres, or the momentum alone, gives polarisation results consistent with those obtained using the purely calorimetric electron energy measurement.

The systematics in the polarisation are different if one uses the ECAL wires or towers for the energy measurement. The two readouts each have an energy scale calibration done with $e^{+} e^{-} \rightarrow e^{+} e^{-}$events. The wire energy scale is known to $0.12 \%$ for $45 \mathrm{GeV}$ electrons, while the tower scale is known to $0.2 \%$. Both measurements suffer from wire response saturation at the center of high energy showers. To account for the induced nonlinearity the measured energy $E_{M E A S}$ must be corrected by adding $(7.8 \pm 0.6) 10^{-4} \times E_{M E A S}^{2}\left(\mathrm{GeV}^{2}\right)$. This coefficient was derived from test beam data and has been checked using $\mathrm{Z}$ decays. The tower-based energy estimator has an additional uncertainty from energy deposits at the edge of the shower which are below the 30 $\mathrm{MeV}$ threshold. The correction for this effect is measured using $\gamma \gamma \rightarrow e^{+} e^{-}$events. It amounts to adding $(0.12 \pm 0.03) \times \sqrt{E_{M E A S}}\left(\mathrm{GeV}^{1 / 2}\right)$ to the measured energy.

Systematics associated with electron identification efficiency and pion misidentification are measured using the kinematically identified electrons and pions from data and Monte Carlo, as explained above. The main tau background is from $\rho \nu$ and the uncertainty in its branching ratio is also taken into account.

The most important non-tau background in $e \nu \bar{\nu}$ is that from $e^{+} e^{-} \rightarrow e^{+} e^{-}$where the $e^{ \pm}$in the recoil hemisphere is misidentified as a pion. The level of this background is estimated by applying the background rejection cuts to events from the high energy kinematically identified $e^{ \pm}$sample in the data and counting the number that have an electron misidentified as a pion. Additionally, the level of $e^{+} e^{-} \rightarrow e^{+} e^{-}$background is seen to be well simulated at each step in the analysis. The uncertainty of the level of this background is limited by the statistics of the misidentified electrons in the kinematically identified sample. As a check, the amount of this background has been allowed to float in the fit for the polarisation. The level of background found in this way is the same as that predicted by the simulation and that computed from the kinematically identified sample.

\subsection{Polarisation with $\mu \nu \bar{\nu}$}

This channel is defined as a hemisphere with one charged track identified as a muon and no reconstructed $\pi^{0}$ s. The two main non-tau backgrounds are $Z \rightarrow \mu^{+} \mu^{-}$and $\gamma \gamma \rightarrow$ $\mu^{+} \mu^{-}$. These backgrounds are suppressed by exploiting their kinematic properties: $Z \rightarrow \mu^{+} \mu^{-}$events produce two back-to-back muons each with energy normally above $90 \%$ of the beam energy, while the muons from $\gamma \gamma \rightarrow \mu^{+} \mu^{-}$usually have less than $10 \%$ of the beam energy, and their acollinearity is typically below $160^{\circ}$. The two analyses place requirements on energy and acollinearity at these levels; stricter conditions on the same quantities are applied when there is an identified muon in the hemisphere opposite the $\tau \rightarrow \mu \nu \bar{\nu}$ candidate.

The LM and NN methods follow different strategies for the estimation of the $Z \rightarrow$ $\mu^{+} \mu^{-}$contribution. In the case of the NN method, the fit range is limited to $0.95 E_{\text {beam }}$ 
to reduce sensitivity to this background, and the remaining background is normalized to the $Z \rightarrow \mu^{+} \mu^{-}$events above $0.95 E_{\text {beam }}$. This leads to a $20 \%$ variation of the level of this background to estimate the systematic uncertainty on the polarisation $( \pm 0.008)$ from this source. In the LM method, the background contribution is taken from the Monte Carlo simulation, and the systematic uncertainty of \pm 0.011 on the polarisation is estimated by letting its contribution float in the fit. Two-photon processes $\gamma \gamma \rightarrow \mu^{+} \mu^{-}$ are considered separately from $Z \rightarrow \mu^{+} \mu^{-}$and contribute much less to the uncertainty on the polarisation.

Tau background is due to the misidentification of pions from hadronic tau decays. The uncertainties on the polarisation are evaluated by varying the amount of background by the uncertainty in the corresponding tau branching ratio plus one statistical standard deviation from the number of misidentified pions in the kinematically identified sample. The corresponding uncertainty on the polarisation is limited by the number of kinematically identified pions. This leads to a $25 \%(10 \%)$ variation of the amount of $\tau \rightarrow \pi \nu\left[n \pi^{0}\right](n \geq 0)$ in the $\mu \nu \bar{\nu}$ sample at high $x$ in the NN (LM) analysis.

The momentum calibration is done as a function of the polar angle using $Z \rightarrow \mu^{+} \mu^{-}$ events. The systematic uncertainty of \pm 0.010 on the polarisation corresponds to the statistical uncertainty in the correction and its possible variation as a function of time, and azimuthal and polar angles.

\subsection{Polarisation with $\pi \nu$}

A pion hemisphere contains one charged track identified as a pion and no photons, either converted or reconstructed in the ECAL. Tracks going through ECAL cracks are rejected in LM but kept by $\mathrm{NN}$ if the $\mathrm{dE} / \mathrm{dx}$ measured in the TPC for the track is less than 1.5 standard deviations above the expectation for a pion of that momentum. Presence of one or more 'fake' photons, as discussed in section 3.2, does not eliminate a candidate $\pi \nu$ hemisphere, as long as no pair of them forms a $\pi^{0}$ mass. The pion channel contains $K \nu$ as well. The kaons have the same kinematics as the pion except at very low energy. Conditions on the recoil hemisphere or on correlations between hemispheres are set, much as in the $\mu \nu \bar{\nu}$ channel, to reduce the contaminations from $e^{+} e^{-} \rightarrow e^{+} e^{-}, e^{+} e^{-} \rightarrow \mu^{+} \mu^{-}$and $\gamma \gamma \rightarrow \mu^{+} \mu^{-}$.

The kinematically identified electrons and muons are used to measure uncertainties from the non-tau backgrounds as well as the backgrounds from misidentified $\tau \rightarrow e \nu \bar{\nu}$ and $\mu \nu \bar{\nu}$. The corresponding variations are $10 \%$ for $e^{+} e^{-} \rightarrow e^{+} e^{-}$background and $20 \%$ for $\gamma \gamma \rightarrow \mu^{+} \mu^{-}$. Backgrounds from $Z \rightarrow \mu^{+} \mu^{-}$and $\gamma \gamma \rightarrow e^{+} e^{-}$are negligible.

The three main sources of tau background are $\mu \nu \bar{\nu}, K^{*} \nu$ and $\pi+n \pi^{0} \nu[n \geq 1]$, and they contribute roughly equally to the systematic uncertainty listed as tau background in table 2. Backgrounds from misidentified $\mu \nu \bar{\nu}$ decays are varied by an amount corresponding to the uncertainty derived from the particle misidentification studies and the uncertainty on the branching ratio. Contamination from $K^{*} \nu\left(K^{*} \rightarrow K_{L}^{0} \pi\right)$ is reduced in the LM analysis by cutting on the calorimeter energy when there is activity in the back part of the HCAL. The systematics due to contamination by tau hemispheres containing photons have been estimated by varying the cuts on energy and distance 
which define fake photons, as well as by varying branching ratios. The resulting change in background is equivalent to varying the $\pi+n \pi^{0} \nu$ contamination by about $20 \%$.

One of the systematics which has been improved is related to charged particle identification. A dependence of the pion identification efficiency with momentum, different in Monte Carlo and data, was mentioned in section 3.1. The resultant corrections to the polarisation for the 1992 data are $0.005 \pm 0.004$ for the LM method and $0.003 \pm$ 0.006 for NN. In the preceding ALEPH paper [2], this effect was not corrected and the systematic uncertainty assigned was the measured bias plus its statistical error.

\subsection{Polarisation with $\rho \nu$}

Candidate hemispheres for the $\rho \nu$ channel have a single charged track and a $\pi^{0}$, which together have invariant mass in the range 0.5 to $1.2 \mathrm{GeV}$. The two types of candidate hemispheres, distinguished by the way in which the $\pi^{0}$ is reconstructed, were discussed in section 3.2. No particle identification is applied to the charged track accompanying a resolved $\pi^{0}$ in the NN analysis; a loose one is applied in the LM analysis. In the case of a merged $\pi^{0}$ the accompanying charged particle is required to be identified as a pion.

In order to reduce sensitivity to differences in fake photon production in data and Monte Carlo, the LM analysis allows a maximum of three photons. The photons which are not part of the $\pi^{0}$ are required to satisfy loosened criteria for fake photons. Thus the LM analysis has higher efficiency than the NN analysis, which allows exactly one or two photons, but LM also has more background from misidentified tau decays. The polarisation obtained by the NN analysis is also stable against extra photons; as a check, it is found to change by $-0.005 \pm 0.005$ (statistical) when an extra photon is allowed in addition to the $\pi^{0}$.

Non-tau background is kept below $0.2 \%$. The mass of the recoil hemisphere is required to be less than $2 \mathrm{GeV}$ to remove $Z \rightarrow q \bar{q}$. Events from two-photon processes are removed by requiring the acollinearity angle to be less than $168^{\circ}$. Hemispheres with an electron in the recoil hemisphere with energy above $95 \%$ of the beam energy are also removed to reduce $e^{+} e^{-} \rightarrow e^{+} e^{-}$background.

One difficulty in reconstructing $\tau \rightarrow \rho \nu$ is the absence of a pure sample of $\pi^{0} \mathrm{~s}$ with which to test the reconstruction algorithms. Thus the tests of photon and $\pi^{0}$ reconstruction are restricted here to tests of consistency between data and Monte Carlo.

One check is to extract the polarisation separately for the rhos with merged $\pi^{0} \mathrm{~s}$ and those with resolved $\pi^{0}$ s. To compare the polarisation extracted using these two samples tests the reconstruction efficiency in different parts of the decay phase space enriched in opposite polarisations. The values found are consistent at the level of 1.6 statistical standard deviations.

A stronger check is to divide the rho sample into the candidates with the charged pion forward and backward in the rho rest frame. These samples should have nearly identical intrinsic polarisations but very different behavior in the detector. The polarisations extracted from the two samples agree to better than one standard deviation. 
To estimate a systematic uncertainty on the polarisation due to photon reconstruction the minimum distance and energy requirements for rejecting fake photons have been changed in steps of $1 \mathrm{~cm}$ and $100 \mathrm{MeV}$ away from the default values of $2.5 \mathrm{~cm}$ and $250 \mathrm{MeV}$ up to $20 \mathrm{~cm}$ and $3 \mathrm{GeV}$. Problems with photon reconstruction and energy estimation associated with clustering and fake photons are the most prominent for low energy photons which are close to the charged track impact point. So by restricting the polarisation measurement to a sample of relatively high energy photons far from the charged tracks such problems may be avoided. The polarisation is generally stable in these variations except that it changes by about 0.01 as the distance cut passes through $6 \mathrm{~cm}$. The statistical significance of the change is about one standard deviation. A change of similar size occurs when the minimum energy passes through $700 \mathrm{MeV}$. The full change from energy and distance is taken as the systematic uncertainty on the polarisation due to photon reconstruction.

Background from misidentified tau decays comes mostly from $\tau \rightarrow \pi+n \pi^{0} \nu[n \geq 2]$. It enters partly through the uncertainty on tau branching ratios, but mostly through $\pi^{0}$ reconstruction, described above.

Energy scales and corrections also contribute to the systematic uncertainty. The largest of these comes from corrections to photon energies for the fraction of the energy deposited outside the central part of the shower in ECAL. These uncertainties have been estimated for the resolved $\pi^{0}$ sample by changing the photon reconstruction parameters while maintaining the average $\pi^{0}$ mass consistent with its known mass. The uncertainty in corrections to all photon energies (including the merged $\pi^{0}$ ' $\mathrm{s}$ ) is similar to the clustering correction uncertainty encountered in the $e \nu \bar{\nu}$ channel for the ECAL towers $\pm 0.03 \times \sqrt{E_{M E A S}}\left(\mathrm{GeV}^{1 / 2}\right)$, and the uncertainty on the polarisation from this source is the dominant energy-related systematic in the $\rho \nu$ channel. The ECAL energy scale and saturation uncertainties introduce much smaller systematics, as does the charged track momentum scale uncertainty. The full uncertainty due to energy measurement is \pm 0.007 .

\subsection{Polarisation with $a_{1} \nu$}

Candidate hemispheres for $\tau \rightarrow a_{1} \nu$ have three tracks, none of which is identified as an electron, and no photons. Since the $a_{1}$ decays through $\rho \pi$, one $\pi^{+} \pi^{-}$pair is required to have invariant mass in the range 0.6 to $1.0 \mathrm{GeV}$. Finally, the reconstructed 3 -pion mass must be below the tau mass.

Particle identification enters the $a_{1}$ analysis only in the rejection of hemispheres with one charged track and a converted photon which was not recognized as such by the conversion-finding algorithm. Photon reconstruction is necessary only to reject $\tau \rightarrow 3 \pi \pi^{0} \nu$ decays; variation of the photon reconstruction parameters leads to a systematic of \pm 0.002 . Pattern recognition problems with close tracks may contribute an uncertainty on the polarisation. They have been studied by comparing the distributions of the angle between the same-sign tracks in 3 -prong tau decays in data and simulation.

The $Z \rightarrow q \bar{q}$ background in the $a_{1}$ comes from the tail of the $q \bar{q}$ fragmentation. It 
is found to be negligible by comparing the distributions of the opening angle between charged tracks for recoil hemispheres in data and simulation.

Three-prong tau decays not coming from the $a_{1}$ are the main background: $\tau \rightarrow K^{*} \nu$, $3 \pi \pi^{0} \nu, K K \pi \nu$, and $K \pi \pi \nu$. The levels of these modes have been varied by an amount corresponding to the difference between the branching ratio used in the simulation and the current world average plus one standard deviation. The only other systematic uncertainties of significance are Monte Carlo statistics and model dependence. Our evaluation of the effect of model dependence on the polarisation has not changed since publication of [2] and the systematic uncertainty of \pm 0.012 is carried over.

\subsection{Average polarisation in the individual decay channels}

The average polarisations for the five decay modes have been combined with the similar results published previously by ALEPH [2]. To combine the results, the systematic uncertainties, Monte Carlo statistics excluded, have been treated as maximally correlated between the present work and the previous publication. Different Monte Carlo data sets were used for the present work and the previously published results. The results are shown in table 5 . The measurements are consistent channel to channel with a $\chi^{2}$ per degree of freedom of 0.9 for the statistical uncertainties only, and 0.6 if the systematics are also taken into account under the assumption of independence from channel to channel.

The tau polarisation has been extracted assuming pure $\mathrm{V}-\mathrm{A}$ couplings in the decay. The systematic uncertainty related to this assumption can be estimated using the measurements of the tau neutrino helicity and the leptonic tau decay parameters [8, 9]. It amounts to about \pm 0.005 on the asymmetry parameter $\mathcal{A}_{\tau}$. The results in this and the following sections do not include this uncertainty.

Radiation in tau decay in the semi-hadronic channels is a potential source of systematic uncertainty. For the most important channels, $\pi \nu$ and $\rho \nu$, the associated systematic uncertainties have been estimated using recent theoretical input [10] by ALEPH and the other LEP [3] experiments to be below \pm 0.001 on $\mathcal{A}_{\tau}$, and negligible for $\mathcal{A}_{e}$.

\begin{tabular}{|l|r|}
\hline decay channel & $1990-1992 \mathrm{ALEPH}$ \\
\hline$e \nu \bar{\nu}$ & $-0.200 \pm 0.051 \pm 0.031$ \\
\hline$\mu \nu \bar{\nu}$ & $-0.124 \pm 0.041 \pm 0.021$ \\
\hline$\pi \nu$ & $-0.142 \pm 0.020 \pm 0.011$ \\
\hline$\rho \nu$ & $-0.108 \pm 0.019 \pm 0.018$ \\
\hline$a_{1} \nu$ & $-0.135 \pm 0.035 \pm 0.020$ \\
\hline
\end{tabular}

Table 5: Average polarisation for the five channels for the 1990-1992 data set. The previously published results [2] and the new results on the 1992 data have been combined assuming the systematic uncertainties are maximally correlated. 


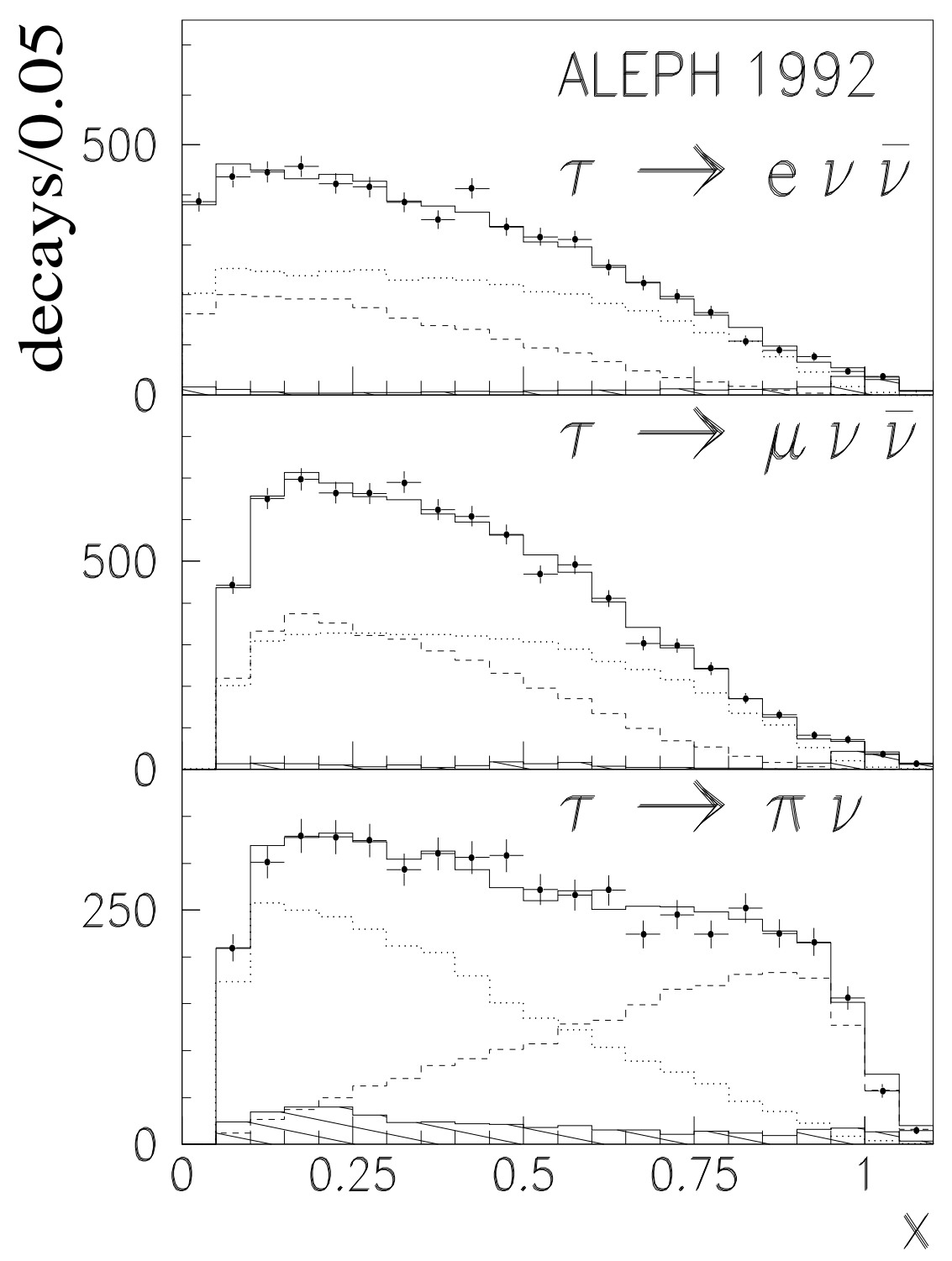

Figure 1: Distributions of $x$ for the electron, muon and pion channels. The data are shown by plotted points with statistical error bars, the individual contributions from the $\sigma_{R}$ and $\sigma_{L}$ simulation by dashed and dotted lines, respectively. The full simulated background, from tau and non-tau sources, is superimposed as a hatched histogram. The solid-line histogram shows the sum of all simulated contributions. 


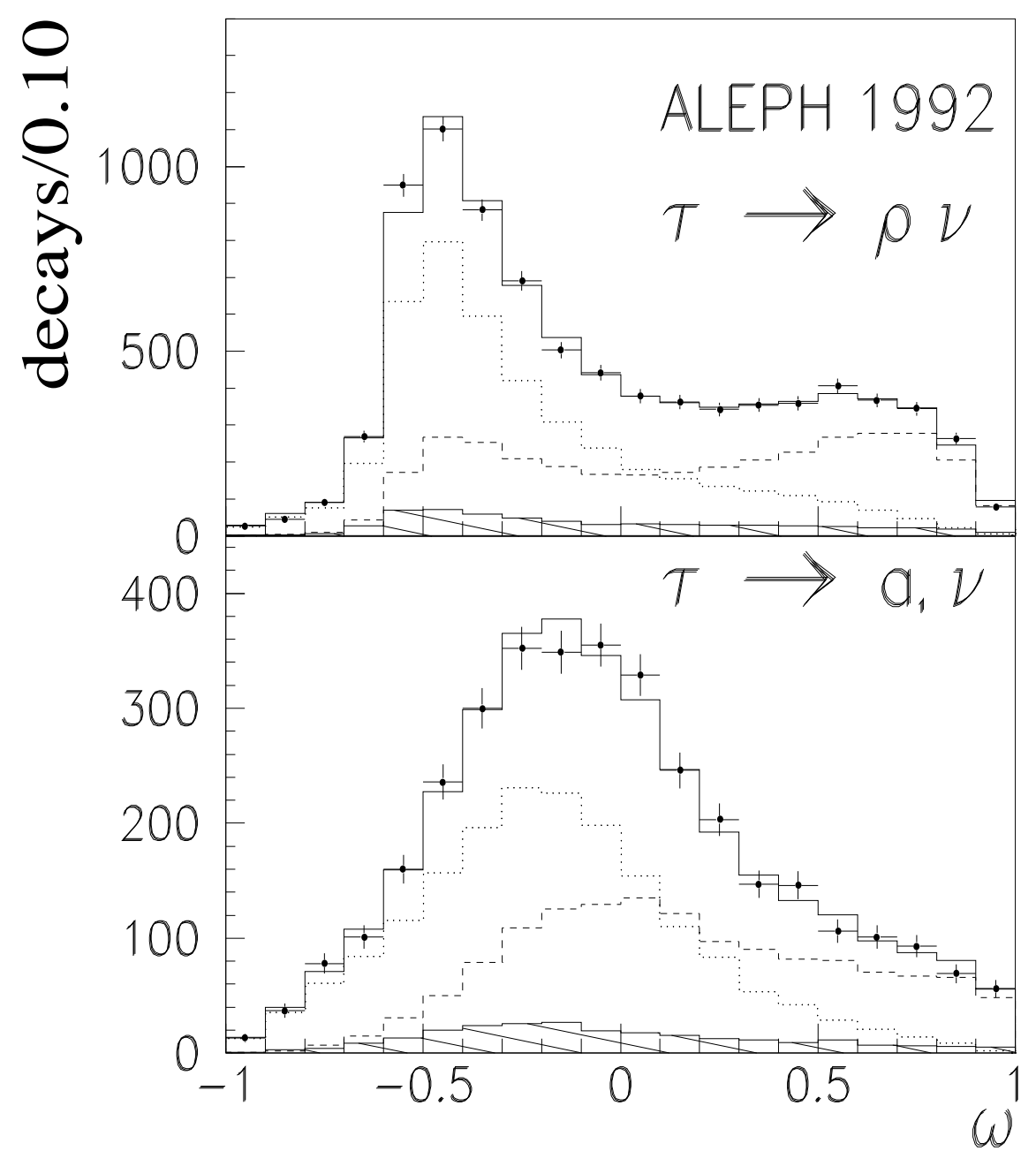

Figure 2: Distributions of $\omega$ for the rho and $a_{1}$ channels. The data are shown by plotted points with statistical error bars, the individual contributions from $\sigma_{R}$ and $\sigma_{L}$ simulation by dashed and dotted lines, respectively. The full simulated background, from tau and non-tau sources, is superimposed as a hatched histogram. The solid-line histogram shows the sum of all simulated contributions. 


\section{Measurement of $\mathcal{A}_{e}$ and $\mathcal{A}_{\tau}$}

The expected dependence of the $\tau$ polarisation on the polar angle $\theta$ between the $e^{-}$ beam and the $\tau^{-}$is given in the Born approximation by equation (2) as a function of the asymmetry parameters $\mathcal{A}_{e}$ and $\mathcal{A}_{\tau}$.

To extract these parameters from the data, the polarisation $P_{i}^{c}$ has been measured in 9 bins of $\cos \theta(i=1,9)$ for the 5 decay channels $(c=1,5)$. Denoting the systematic error of the channel $c$ by $\sigma_{s}^{c}$, and the statistical error on the measurement in the bin $i$ of channel $c$ (including Monte Carlo statistics) by $\Delta_{i}^{c}$, the $\chi^{2}$ is written

$$
\chi^{2}=\sum_{c}\left[\left(\frac{\delta^{c}}{\sigma_{s}^{c}}\right)^{2}+\sum_{i}\left(\frac{P_{i}^{c}-P_{\tau}\left(\cos \theta_{i}, A_{e}, A_{\tau}\right)-\delta^{c}}{\Delta_{i}^{c}}\right)^{2}\right],
$$

where $\delta_{c}$ are systematic offsets for each channel and $P_{\tau}\left(\cos \theta_{i}, A_{\epsilon}, A_{\tau}\right)$ is from equation (2). This equation does not include photon exchange or the effect of initial state radiation and so results obtained using it must be corrected slightly. In order to avoid confusion, the quantities derived directly from the fit are written $A_{e}$ and $A_{\tau}$ while the corrected quantities, which are related to $g_{V e} / g_{A e}$ and $g_{V \tau} / g_{A \tau}$ by equation (3), are written $\mathcal{A}_{e}$ and $\mathcal{A}_{\tau}$.

The asymmetry parameters and their errors are obtained by minimizing the $\chi^{2}$ with respect to the 7 parameters $A_{\epsilon}, A_{\tau}$, and $\delta^{c}(c=1,5)$. The offsets are found to be consistent with zero. The correlation coefficient of $A_{e}$ and $A_{\tau}$ is $5.3 \%$. The fit assuming $e-\tau$ universality yields $A_{\epsilon-\tau}$. In the following results the Monte Carlo statistical errors have been transferred to the systematics.

The systematic errors have been treated as uncorrelated between channels and totally correlated between bins for a given channel, except for the electron channel where the Bhabha background is concentrated in the forward bin. The lack of channel to channel correlation is clear from table 2: the leptonic channels have large contributions from different sources of non-tau background (which are mostly absent in the semi-hadronic channels). The $\rho$ is dominated by uncertainties in $\pi^{0}$ reconstruction, the $a_{1}$ by model dependence. The correlation between bins is due to the fact that the systematics are independent of $\theta$ and are estimated globally. Few sources of systematic uncertainties are correlated across channels; the momentum scale and particle (mis)identification are the two most important ones. The correlated effects contribute an additional uncertainty of less than \pm 0.0015 to the full systematic uncertainty on $\mathcal{A}_{\tau}$, which is \pm 0.009 assuming no channel to channel correlation.

The reduction in statistical power due to the correlation between the helicities of the two hemispheres of an event has been evaluated to be $4 \%$ and it has been taken into account for the results below.

The contributions to the systematics on $A_{e}$ from the apparatus, such as sagitta distortions in the TPC, and Bhabha background which could generate effects asymmetric in the sign of charge and $\cos \theta$ have been evaluated and found to be negligible $( \pm 0.001$ and \pm 0.001$)$. Including all these effects, the results of the fit for the 1992 data are $A_{\tau}=0.130 \pm 0.016 \pm 0.009, A_{e}=0.133 \pm 0.021 \pm 0.007$, and $A_{e-\tau}=0.131 \pm 0.013 \pm 0.006$. 


\section{Interpretation and Results}

To interpret these measurements in terms of the $\mathrm{Z}$ couplings to the electrons and taus one must first correct them for the effects of the photon exchange and initial state radiation. Initial state radiation effectively changes the centre of mass energy available for $e^{+} e^{-}$annihilation. The photon contribution slightly dilutes the polarisation of the taus that come from $\mathrm{Z}$ decay and modifies the angular dependence.

The Standard Model program ZFITTER [11] has been used to calculate the tau polarisation in bins of polar angle including the effects mentioned above. Those Standard Model "data" are then fit using equation 2. The differences between the values of $A_{\tau}$ and $A_{e}$ resulting from the fit and those of $\mathcal{A}_{\tau}$ and $\mathcal{A}_{e}$ calculated using the effective couplings provided by ZFITTER are the basis for the correction applied to the data. The full correction at the $Z$ peak amounts to adding 0.003 to both $A_{\tau}$ and $A_{e}$

The corrected results for the 1992 data are $\mathcal{A}_{\tau}=0.133 \pm 0.016 \pm 0.009, \mathcal{A}_{e}=$ $0.136 \pm 0.021 \pm 0.007, \mathcal{A}_{e-\tau}=0.134 \pm 0.013 \pm 0.006$. The new results have been combined with the published ALEPH data [2]. The results for data taken in 1990, 1991, and 1992 are

$$
\begin{gathered}
\mathcal{A}_{\tau}=0.136 \pm 0.012 \pm 0.009 \\
\mathcal{A}_{e}=0.129 \pm 0.016 \pm 0.005 \\
\mathcal{A}_{e-\tau}=0.134 \pm 0.010 \pm 0.006
\end{gathered}
$$

To visualize the results as a function of $\cos \theta$ the channel measurements for all ALEPH data have been combined bin by bin. They are shown, with statistical errors only, in figure 3 . The superimposed curves are from equation 2 using the measured parameters.

The results support the hypothesis of lepton universality in the Z couplings to leptons:

$$
\begin{gathered}
g_{V \tau} / g_{A \tau}=0.0685 \pm 0.0076, \\
g_{V e} / g_{A e}=0.0650 \pm 0.0086, \\
\frac{g_{V \tau} / g_{A \tau}}{g_{V e} / g_{A e}}=1.05 \pm 0.17 .
\end{gathered}
$$

In terms of the effective weak mixing angle or $g_{V l} / g_{A l}$ the results are

$$
\begin{aligned}
g_{V l} / g_{A l} & =0.0673 \pm 0.0058 \\
\sin ^{2} \theta_{W}^{e f f} & =0.2332 \pm 0.0014
\end{aligned}
$$




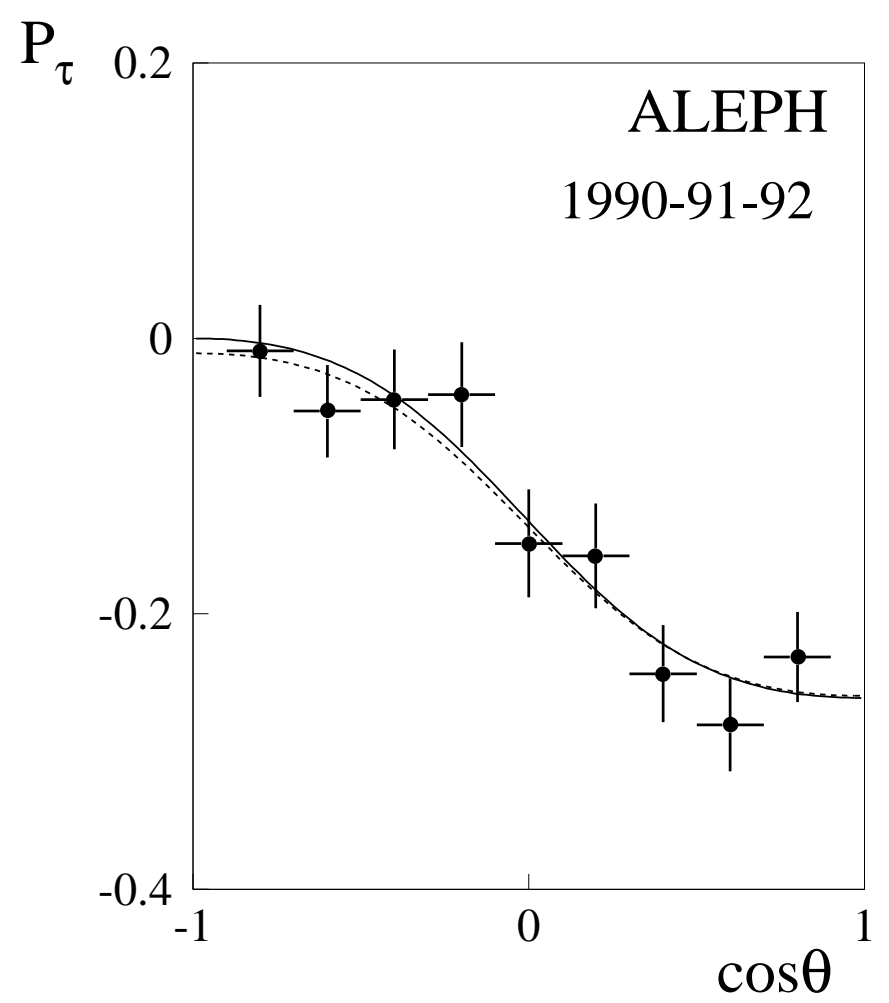

Figure 3: Polarisation dependence on $\cos \theta$ for data taken in 1990, 1991, and 1992. The dashed line shows the result of the fit without the universality constraint, the solid line the result of the fit with the constraint.

\section{Acknowledgements:}

We wish to thank the CERN accelerator divisions for continued good operation of the machine and the engineers and support people at our home institutes without whom this work would be impossible. Those of us from non-member states would like to thank CERN for its hospitality and support.

\section{References}

[1] ALEPH Collab., D. Decamp et al., Phys. Lett. B265 (1991) 430.

DELPHI Collab., P. Abreu et al., Z. Phys. C55 (1992) 555.

L3 Collab., O. Adriani et al., Phys. Lett. B294 (1992) 466.

OPAL Collab., G. Alexander et al., Phys. Lett. B266 (1991) 201.

L3 Collab., O. Acciarri et al., Phys. Lett. B341 (1994) 245.

OPAL Collab., R. Akers et al., Z. Phys. C65 (1995) 1.

[2] ALEPH Collab., D.Buskulic et al., Z. Phys. C59 (1993) 369. 
[3] The LEP Collaborations, Combined Preliminary Data on Z Parameters from the LEP Experiments and Constraints on the Standard Model, CERN preprint CERNPPE/94-187.

ALEPH Collab., D. Buskulic et al., Z. Phys C62, (1994) 539.

Delphi Collab., P. Abreu et al., Nucl. Phys. B418 (1994) 403.

OPAL Collab., R. Akers et al. Z Phys C61 (1994) 357.

L3 Collab., M. Acciarri et al. Z. Phys C62 (1994) 551.

[4] Yung-Su Tsai, Phys. Rev. D4 (1971) 2871.

[5] S. Jadach, Z. Wạs, Z Physics at LEP I, CERN 89-08, eds. G. Altarelli, et al., Vol. 1, p. 235 (1989).

[6] S. Jadach, J.H. Kühn and Z. Was, Comp. Phys. Comm. 64 (1991) 275.

S. Jadach, B.F.L. Ward and Z. Wạs, Comp. Phys. Comm. 66 (1991) 276.

[7] ALEPH Collab., D.Decamp et al., Nucl. Inst. Meth. A294, (1990) 121.

[8] ALEPH Collab., D. Buskulic et al., Phys. Lett. B321 (1994) 168.

ALEPH Collab., CERN preprint, Michel Parameters and $\tau$ Neutrino Helicity from Decay Correlations in $Z \rightarrow \tau^{+} \tau^{-}$, CERN-PPE/94-209.

[9] ARGUS Collab., H.Albrecht et al., Phys. Lett. B337 (1994) 383.

[10] R. Decker and M. Finkemeier, Phys. Rev. D48 (1993) 4203.

M. Finkemeier, Radiative corrections to the decay $\tau \rightarrow \pi \nu$, Ph.D. Thesis, University of Karlsruhe, Feb. 1994.

[11] D. Bardin et al., ZFITTER: An Analytic Program for Fermion Pair Production in $e^{+} e^{-}$Annihilation, CERN preprint CERN-TH. 6443/92. 